\title{
EL HOGAR EN LOS FILMES DE PIXAR: USO CREATIVO Y SIGNIFICADO
}

\section{HOME IN PIXAR FILMS: CREATIVE USE AND MEANING}

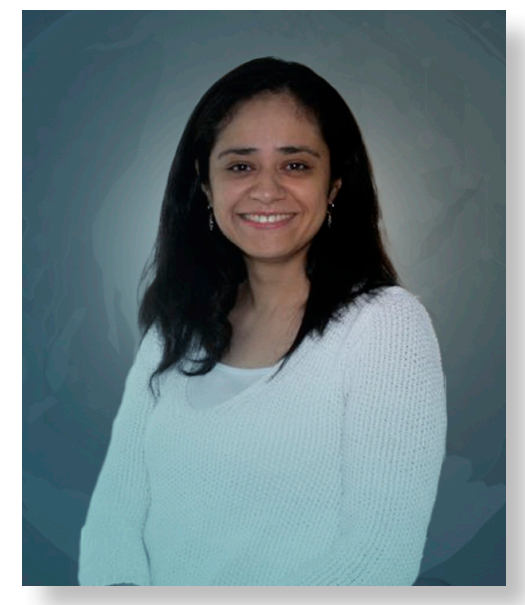

Ana M. Pérez-Guerrero

Doctora en Comunicación Audiovisual por la Universidad Rey Juan Carlos, Madrid

Universidad de La Sabana, Colombia

ana.m.perezguerrero@gmail.com

ORCID: https://orcid.org/0000-0001-9325-965X

Venezuela/España

DOI: DOI: https://doi.org/10.5377/umhs.v2i1.12997

Recibido: 30 de junio de 2021

Aceptado: 16 de noviembre de 2021

desde 1995 hasta 2021, a fin de encontrar el sentido que comunican sus historias sobre este asunto. Para ello, se realiza un análisis textual de estos filmes, en relación a tres dimensiones del hogar, que van desde lo material a lo abstracto: casa, ciudades y tramas, que definen qué es una patria y en dónde radica el corazón de los personajes. Finalmente, se concluye en que el sentido de hogar en este estudio cinematográfico recae en una noción de familia, abierta y cambiante, en el que el valor del trabajo como pasión y realización del héroe al servicio de otros tiene una importancia crucial. Una visión creativa que contribuye a la longevidad de las historias y defiende la vigencia de las acciones positivas para la construcción de la sociedad.

PALABRAS CLAVES: Pixar, representación del hogar, cine familiar, cine de animación, familia.

\section{RESUMEN}

El cine de Pixar es conocido mundialmente como sinónimo de cine familiar de calidad. Sus películas, como muchas cintas de Hollywood orientadas a este público, emplean tramas clásicas de retorno al hogar. Sin embargo, el hogar en estas producciones no ha generado trabajos académicos que aborden de manera integral el concepto, al tratar solo la estructura narrativa o su representación desde algunas de sus facetas, y casi siempre se aborda desde los estudios culturales y de género, y no abarcan todos los títulos disponibles de esta compañía. El presente artículo tiene como objeto la exploración de las estrategias narrativas que Pixar emplea para retratar el hogar en sus largometrajes,

\section{ABSTRACT}

Pixar films is known worldwide as a synonym for quality family cinema. Its movies, like others from Hollywood, aimed at this audience, employ classic homecoming plots. However, the home in these productions has not generated academic works that comprehensively address the concept, treating only the narrative structure or its representation from some of its facets, and many times these papers are approached from cultural and gender studies, and they do not cover all titles available from this company. The purpose of this article is to explore the narrative strategies that Pixar uses to portray the home in its feature films, from 1995 to 2021, to find the meaning on this matter 
that their stories communicate. For this, a textual analysis of these films is carried out, in relation to three dimensions of the home, ranging from the material to the abstract: house, cities and plots, which define what a homeland is and where the hearts of the characters lie. Finally, it is concluded that the sense of home in this film study falls on a notion of family, open and changing, in which the value of work as a passion and fulfillment of the hero at the service of others is crucial. A creative vision that contributes to the longevity of the stories and defends the validity of positive actions for the construction of society.

KEYWORDS: Pixar, representation of the home, family cinema, animation cinema, family.

\section{INTRODUCCIÓN}

Los filmes de Pixar han sido aclamados consecutivamente por crítica y taquilla, desde el lanzamiento de su primer largometraje, Toy Story (John Lasseter, 1995), hasta la fecha. La extraordinaria factura y fuerza emocional de sus historias han convertido su filmografía en sinónimo de cine familiar de calidad. Por otra parte, el hogar es de los espacios más importantes en los que se desarrolla la narrativa clásica de aventuras, en las que se adscriben estas películas, no solo por representar el territorio en el que se desarrollan las fases de alteración y ajuste en la mayoría de sus tramas, sino también por constituir una metáfora sobre la identidad del héroe (Kuntz, 1993). No en vano se trata del lugar al que él pertenece, en el que está su corazón y al que desea regresar. Un aspecto que dibuja un "marco que se mueve de lo literal a lo abstracto" (Naficy, 1999, p. 6), según sea el tratamiento creativo de los términos con lo que se le asocia: casa, ciudad, familia, seres queridos, patria, y que facilitan la expresión de los valores del relato.

Si bien el hogar en los filmes de Pixar es un tema que ha despertado el interés de varios académicos, es llamativa la preponderancia de trabajos abordados desde los estudios culturales y de género, que adoptan de manera casi exclusiva una dimensión del concepto relacionada con la familia y su representación (Jenkings, 2015), o con las viviendas, asociándolas a cuestiones de carácter ideológico (Treinblay, P., 2011; Cheu, 2013; Meinel, 2014; Sperb, 2015; Holliday, 2016); abandonando, hasta cierto punto, otras nociones y otras posibles perspectivas de análisis, como la de la creación y la narración, que podrían arrojar más luz sobre la construcción de los discursos de estos relatos, siendo la excepción el trabajo de Prokhorov (2021), quien aborda en algunos filmes de Pixar tres tipos de metáforas empleadas en el camino del héroe en esas tramas. Así, cabe preguntarse ¿cuáles son las características de los hogares en la filmografía de Pixar? ¿Dónde está el corazón de sus héroes? En definitiva, ¿qué se puede deducir de los usos creativos que se da al hogar en la narración de los veinticuatro títulos estrenados por esta compañía cinematográfica desde 1995 a 2021 ?

El presente artículo tiene como objeto de estudio las estrategias narrativas y creativas que Pixar emplea para retratar el hogar en sus producciones, a fin de encontrar el sentido que comunican sus historias sobre este asunto. Para conseguir este propósito, expone, en primer lugar, en qué consiste el instrumento de análisis empleado para abordar los filmes de esta productora. A continuación, da paso al análisis y discusión de resultados, en que el sentido de hogar en este estudio cinematográfico recae en una noción de familia, abierta y cambiante, en el que el valor del trabajo como pasión y realización del héroe 
al servicio de otros tiene una importancia crucial.

\section{METODOLOGÍA}

No hay representación o discurso audiovisual que se pueda relatar sin un escenario dotado de características espaciales concretas, que contribuyan a la narración de la pieza (Cauqueling, 2008), a partir de su relación con los personajes y la acción que en ella se desarrolla. Dichos rasgos espaciales, como afirma Lefevre (2006), aunque tangibles y precisos, acogen espacios más grandes, que pueden ser invisibles o abstractos, y cuya relevancia solo se establece mediante la interpretación, en el contexto del propio relato.

Puesto que "el hogar se refiere a un lugar imaginario de pertenencia, a una casa de concreto, que brinda protección y comodidad, o a una comunidad geocultural concreta" (Bronfen, 2004, p. 22), este trabajo estudia las manifestaciones materiales del hogar, tanto en espacios privados como públicos con los que los personajes puedan sentirse íntimamente vinculados. Del mismo modo, aborda dimensiones más abstractas, como la patria, en las tramas de los filmes que integran el corpus de investigación. A saber: la tetralogía de Toy Story (John Lasseter,1995, 1999; Lee Unkrich, 2010; Josh Cooly, 2019); A Bug's Life (John Lasseter \& Andrew Stanton, 1998); Monsters, Inc. (Pete Docter, Lee Unkrich \& David Silverman, 2001); Monsters University (Dan Scanlon, 2013); Finding Nemo (Andrew Stanton \& Lee Unkirch, 2003); Finding Dory (Andrew Stanton \& Angus McLane, 2016); The Incredibles 1 y 2 (Brad Bird, 2004, 2018); la trilogía de Cars (Lasseter \& Ranft 2006, Lasseter \& Brad Lewis 2011 y Brian Free, 2017); Ratatouille, (Brad Bird, 2007); Wall-E (Andrew Stanton, 2008), Up (Pete Docter, Bob Peterson, 2009); Brave (Brenda Chatman, 2011); Inside Out, (Pete Docter \& Ronie del Carmen, 2015);
The Good Dinosaur, (Bob Peterson, 2015); Coco (Lee Unkrich, 2017); Onward (Dan Scanlon, 2019); Soul (Pete Docter \& Kemp Powers, 2020) y Luca (Enrico Casarosa, 2021).

Esta selección excluye los cortometrajes del Estudio, por considerar que su relevancia no alcanza la popularidad y reconocimiento de los largometrajes, cuyo contenido es mucho más representativo y popularmente identificado con la línea editorial de Pixar/Disney, máxime tras el lanzamiento exclusivo de sus dos últimos títulos en la plataforma Disney+, desagregando el habitual visionado de los cortos como preludio de sus filmes. En consecuencia, esta elección parece la más adecuada para responder a la pregunta de investigación aquí planteada.

Dado el carácter cualitativo de esta indagación, se diseña un instrumento de análisis narratológico, aplicable a las tres dimensiones del hogar que se pretende estudiar (casa, ciudad y trama). En primer lugar, para el abordaje de las viviendas se emplean los conceptos propuestos por Gastón Bachelard (1994) en Poética del espacio, por proveer una perspectiva fenomenológica del uso que se da a las moradas por parte de los personajes, a lo que se añade el modelo de análisis de Rampazzo Gambarato (2010) para el estudio de los objetos que decoran las estancias, a partir de un criterio de funcionalidad, que permita entender su uso escenográfico y expresivo. En segundo lugar, para el estudio de la patria, en su concepción más básica y material, se aborda el diseño y función de ciudades y espacios exteriores, para lo que se toma como referencia los términos de Casetti y Di Chío (1996), que permiten la caracterización de la ambientación y su función narrativa. Por último, para el estudio de la patria, en un sentido más abstracto, se concreta la ubicación del hogar en la estructura narrativa, para lo que se apela a las posibilidades de hallazgo o renuncia de este en 
las tramas de aventuras, propuestas por SánchezEscalonilla (2009).

\section{FASES DEL TRABAJO}

En cuanto al procedimiento, se realiza el análisis narratológico de las casas en los títulos de Pixar en los que se representan las residencias personales de sus protagonistas, lo que obliga a excluir de la muestra, solo para esta fase del estudio, la trilogía de Cars y A Bug's Life, ya que en ellas sus protagonistas no tienen una vivienda personal propiamente dicha. La idea de esta parte del trabajo es identificar la función narrativa de estas viviendas, teniendo en cuenta su diseño, el tipo de acciones que se desarrollan en ellas, contrastándolas con otros espacios de la historia. No en vano, los espacios dramáticos están constituidos por subespacios que se oponen entre sí, expresando el conflicto argumental (Ubersfeld, 1993).

En primera instancia, se estudia el diseño exterior de las casas, como la forma en que el personaje se presenta ante el mundo. En segundo lugar, se aborda el empleo que los personajes hacen de sus moradas, para reflejar de manera clara su conflicto interno, amén de expresar la riqueza de su vida interior.

Estos usos pueden ser identificados, en términos de Bachelard (1994), como nidos, cuyo énfasis se relaciona con el bienestar, confort y ensoñación de sus habitantes, mientras que el morador puede entender su hogar como una concha que le protege y defiende su soledad e impide cualquier perturbación externa hacia el interior. Estas casas suelen estar diseñadas como auténticas fortalezas o construidas bajo tierra.

En atención a que la riqueza informativa de una vivienda aumenta cuando se traspasan sus puertas, se abordan las dependencias privadas, como expresión de la dimensión emocional del personaje, en su nivel más evidente. Para ello, se explora el uso creativo de elementos que integran la decoración de las casas y, de manera especial, aquellos con los que el personaje se relaciona.

Se sigue un criterio de funcionalidad, el cual puede ser (a) escenográfico al contribuir a la creación y adecuación del escenario en relación a un entorno o (b) semántico, en la que el objeto contribuye a establecer un significado entre él y la imagen en la que aparece, dotando de emociones los espacios y contribuyendo a la red de símbolos del relato. Para este estudio se obvian, además de las cintas antes excluidas, Ratatouille, Finding Nemo y Finding Dory, debido a que las residencias allí representadas atienden solo a la dimensión individual de sus propietarios (peces, ratas $u$ hormigas) $y$ carecen de una decoración que ayude a caracterizar su personalidad.

Para los análisis que siguen se retoma la muestra inicial con todos los filmes de Pixar, ya que se trata de estudiar las ciudades y los espacios a los que el personaje se sienta vinculado, así como las tramas.

En cuanto a las ciudades, se aplica, como se ha dicho, el modelo de Casetti \& Di Chío (1996), para conocer el tratamiento creativo que se dan a esos lugares desde la narración, enfocándose en la singularidad del diseño de estos espacios, definiendo si se trata de ambientes ricos o pobres en detalles; armónicos o disarmónicos; históricos o metahistóricos, en referencia a un periodo determinado, o si solo son lugares típicos que sirven de fondo a la historia o si, por el contrario, son caracterizados, ocupando así una tarea más relevante en la historia. 
Finalmente, se aborda el hogar en la estructura narrativa, ya que este dato resulta de gran interés para determinar su naturaleza en el corazón del relato, al contrastar su representación en las fases de alteración y ajuste.

En la fase de alteración, el hogar se representa como el territorio habitual del personaje, donde vive en un ambiente familiar, con viejos conceptos y patrones emocionales (Campbell, 1993). Como punto de partida de la historia, en esta etapa se compone el contexto y base central de la figura principal del argumento (Vogler, 2007). Es en ella donde se establecen las normas de posibilidad de ese mundo y su nivel de conflicto. Entre otros aspectos, que lo unen a la última fase dramática, aquí se da a conocer los afectos que el protagonista añorará de su casa durante la aventura, sirviendo como base para la revelación final de la entidad de la patria como hogar. Y es que el lugar al que se llega no necesariamente coincide con el sitio de partida; al menos, no en lo referente a espacios y a los personajes que estaban presentes en el primer acto dramático de la trama. Por tanto, en este análisis se indaga si el héroe encuentra su hogar en el mundo extraordinario o en el mundo ordinario; o si más bien, renuncia al hogar hallado en el mundo extraordinario o renuncia al hogar recuperado en el mundo ordinario.

\section{ANÁLISIS DE LOS RESULTADOS}

La casa es el origen del hogar y su expresión física, es el lugar en el que se reside habitualmente con o sin la familia, es el primer recinto del hombre y su universo más personal. Como afirma Bachelard (1994), todas las áreas de una vivienda, desde la fachada a sus habitaciones, le confieren un puesto sin igual, como objeto de estudio de los valores de intimidad; sobre todo, si se atiende al "tiempo a su unidad y a su complejidad, con el fin de integrar todos sus valores particulares en un valor fundamental" (Bachelard, 1994, p. 27).

Si bien las viviendas de los protagonistas de Pixar no tienen el mismo peso dentro de las tramas, por razones narrativas y dramáticas, y que no siempre aparecen solo en el primer y tercer acto dramático, es posible afirmar que, tras contrastar los eventos que ocurren dentro de ella con otros que suceden fuera, la mayoría de estas edificaciones remiten al lugar en el que el personaje se siente protegido, una percepción que condiciona su diseño y su uso.

En cuanto al diseño exterior de estas casas, se puede comprobar, que la mayoría responden a una arquitectura vertical, la cual remite, en términos de Bachelard, (1994) a la ensoñación y a la protección de sus habitantes al destacar los tejados en su diseño. Por ejemplo, Woody y su pandilla se acercan a su “cielo feliz" (Price, 2008, p. 122) en la habitación de Andy y también, al mudarse al cuarto de Bonnie; ambos situados en una segunda planta. Asimismo, en Luca, Alberto puede proteger sus sueños de aventuras y mirar de forma segura el mundo humano desde lo alto de su torre derruida, ubicada en Isola del Mare.

Si se comparan estas viviendas, con las de otros protagonistas, cuyo diseño, responden a una predominancia de las líneas horizontales (Wall-E, Ratatouille, Incredibles 1, Finding Nemo, Finding Dory, The Good Diosaur), se puede afirmar que el empleo de estas viviendas más bajas responde a la dimensión individual de los personajes que las habitan, como en el caso de la anémona de Marlin. Pero también, esta elección, se relaciona con las necesidades concretas de una historia, especialmente, cuando un personaje debe esconderse o protegerse de algo, como Bob Parr, en The Incredibles 1, quien se ve obligado a morar en una casa de un solo piso, 
en una urbanización media estadounidense, para camuflar la identidad de superhéroes de él y de su familia. Al mismo tiempo, este diseño subraya aún más la incomodidad del personaje en una vida que se le queda corta ${ }^{1}$.

Por otra parte, cabe destacar la similitud de los protagonistas que habitan en estas casas o que viven en el fondo del mar o debajo de la tierra. La mayoría de ellos, coinciden en su carácter soñador, por lo que se ven abocados a salir al encuentro de una vivienda más acorde con sus sueños (The Incredibles 1, Ratatouille, Wall-E, Luca) ${ }^{2}$. En este sentido, solo un domicilio vertical de los estudiadas, impulsa a su dueño a lo más alto, la residencia de los Friedricksen $(U p)$.

La morada de Carl, de claras reminiscencias al domicilio de George y Mary Bailey, en It's a Wonderful Life (Frank Capra, 1946), al ser contrastada con los edificios en construcción que la flanquean, resulta una casa minúscula, lo cual tiene todo el sentido cuando se conoce el uso que su dueño hace de ella, como se verá más adelante. Pero, además, la convierte en un símbolo de resistencia que expresa la renuencia del personaje a dejar ir sus recuerdos y a no aceptar la llegada de una nueva época ${ }^{3}$. Y es solo elevándose por encima de los rascacielos, que Carl se reconcilia con la realidad de los tiempos y su casa encuentra su auténtico lugar, en lo alto de las Cataratas Paraíso, donde residen los sueños del matrimonio que la habitó por años y se cumplen las promesas hechas con el corazón.

En definitiva, parece que aun cuando las líneas indiquen lo contrario, las casas de los protagonistas de Pixar tienen una concepción evocadora, que refuerza con claridad las necesidades de la historia y en las que aportan matices de quiénes son estos personajes, a través de los usos que hacen de sus moradas.

En este sentido, se puede afirmar que la mayoría de ellos optan por emplear sus casas como nidos: sitios acogedores, idílicos, en los que habitar, recordar y soñar, incluso si se vive en un camión (Wall-E), en un castillo fortificado (Brave) o se le dé el uso de concha, como Wall-E y Carl en $U p^{4}$. En ellos predominan los principios de afinidad y la luz cálida ${ }^{5}$. Signo de que son espacios en los que les gusta estar, soñar y habitar6.

De hecho, si se comparan estas viviendas con los domicilios fortificados u ocultos de todos los antagonistas, se puede colegir cómo son retrato de las fuerzas en pugna. $Y$ es que las viviendas de los antagonistas, se caracterizan por que su interior resulta frío, como la sofisticada Isla Nomanisa, centro de operaciones de Sindrome (The Incredibles); o tienden a lucir un poco más siniestros, como la cueva de Muntz en las Cataratas Paraiso $(U p)$ e inquietantes como los espacios nocturnos que convierten en cárcel la guardería Sunnyside (Toy Story 3) y los serpenteantes escondrijos de los villanos de Monsters, Inc. y The Incredibles 2, por nombrar algunos ejemplos.

1 Otros personajes que habitan en casas horizontales, son Wall-E, para protegerse de las inclemencias de un planeta enfermo por la contaminación y el exceso.

2 Aquí cabe indicar una excepción en Brave, dado que Mérida, quien vive en una fortaleza (vertical), debe salir de ella y llevar consigo a su madre para propiciar un encuentro entre ellas y restablecer el equilibrio en el reino.

3 Otra referencia de esta vivienda en una situación de desalojo se halla a partir del segundo siete en el videoclip de la canción Thank you de Dido. Ver: https://bit.ly/3liellO

4 De hecho, la casa de los Friedricksen tiene más de un uso en la película, pero, sobre todo, se convierte en un personaje más, con quien su propietario conversa como si se tratara su fallecida esposa.

5 La afinidad remite a un menor contraste entre los componentes visuales: "cuanto mayor es el contraste en un componente visual, más aumenta la intensidad visual o la dinámica. Cuanto mayor es la afinidad en un componente visual, más disminuye la intensidad visual o dinámica” (Block, 2008), lo que subraya el bienestar y agrado que se vive en estos recintos.

6 Un rasgo que, según Heidegger (1989), abarca nuestra existencia como mortales, que implica cuidar. 
Lo anterior no implica que dentro de las ca-

\begin{tabular}{|c|c|c|c|}
\hline \multicolumn{4}{|c|}{ Casas presentadas en los filmes de Pixar } \\
\hline Título & Domicilio & Arquitectura & Usos \\
\hline Toy Story 1 & Casa de Andy & Vertical & Nido \\
\hline Toy Story 2 & Casa de Andy & Vertical & Nido \\
\hline Monsters, Inc. & Casa de Sulley & Vertical & Nido \\
\hline Finding Nemo & Anémona & Horizontal & Nido \\
\hline $\begin{array}{l}\text { The Incredi- } \\
\text { bles }\end{array}$ & $\begin{array}{l}\text { Casa en urba- } \\
\text { nización }\end{array}$ & Horizontal & Nido \\
\hline Ratatouille & $\begin{array}{l}\text { Casa campes- } \\
\text { tre/casa Lin- } \\
\text { güini/balsa }\end{array}$ & $\begin{array}{l}\text { Vertical/ } \\
\text { Horizontal }\end{array}$ & Nido \\
\hline Wall-E & Camión & Horizontal & $\begin{array}{l}\text { Nido/ } \\
\text { concha }\end{array}$ \\
\hline$U p$ & Casa/ Dirigible & Vertical & $\begin{array}{l}\text { Nido/ } \\
\text { concha }\end{array}$ \\
\hline Toy Story 3 & $\begin{array}{l}\text { Casa de Andy/ } \\
\text { Casa de Bon- } \\
\text { nie }\end{array}$ & Vertical & Nido \\
\hline Brave & Castillo & Vertical & $\begin{array}{c}\text { Nido/ } \\
\text { fortaleza }\end{array}$ \\
\hline Inside Out & $\begin{array}{l}\text { Casa San Fran- } \\
\text { cisco/ } \\
\text { Centro de } \\
\text { operaciones }\end{array}$ & Vertical & Nido \\
\hline $\begin{array}{l}\text { The Good } \\
\text { Dinosaur }\end{array}$ & Granja & Horizontal & Nido \\
\hline Finding Dory & $\begin{array}{l}\text { Arrecife / } \\
\text { Instituto de } \\
\text { Biología Ma- } \\
\text { rina }\end{array}$ & Horizontal & Nido \\
\hline Coco & $\begin{array}{l}\text { Casa de Mi- } \\
\text { guel }\end{array}$ & Vertical & Nido \\
\hline $\begin{array}{l}\text { The Incredi- } \\
\text { bles } 2\end{array}$ & $\begin{array}{l}\text { Casa de Dea- } \\
\text { vor }\end{array}$ & Vertical & Nido \\
\hline Toy Story 4 & $\begin{array}{l}\text { Casa de Andy/ } \\
\text { Casa de Bon- } \\
\text { nie }\end{array}$ & Vertical & Nido \\
\hline Onward & Casa familiar & Vertical & Nido \\
\hline Soul & $\begin{array}{l}\text { Apartamento } \\
\text { de Joe }\end{array}$ & Vertical & Nido \\
\hline Luca & $\begin{array}{l}\text { Casa de Al- } \\
\text { berto/ } \\
\text { casa de Giulia }\end{array}$ & Vertical & Nido \\
\hline
\end{tabular}

Nota: elaboración propia. sas de los protagonistas no ocurran acciones emocionalmente álgidas o conflictivas. Sin embargo, estas acciones no son más que el signo de una dinámica familiar cotidiana (The Incredibles, Onward, Inside Out) o son el preludio del encuentro con un nuevo amigo, como ocurre en la escena en la que Mike y Sulley llevan a Boo a su casa, mientras las autoridades de Monstruopolis buscan a la niña o las secuencias en que Spot asusta a Arlo en el granero de su casa (The Good Dinosaur).

\section{La casa, de puertas para adentro}

Las estancias interiores de las casas son un espacio dramático de primer orden que, como se ha visto, puede ser una variable crucial al representar más que la actitud de su morador frente al mundo; reflejan el estado de su alma y su vida emocional, para lo que los objetos que las adornan, contribuyen de manera conjunta y significativa.

\section{a. Las estancias}

“Todo espacio reducido en donde nos gusta acurrucarnos, agazaparnos sobre nosotros mismos, es para la imaginación una soledad, es decir, (...) el germen de una casa" (Bachelard, 1995, p. 127). Así, la distribución de las estancias privadas de las viviendas es muestra de la complejidad y la cosmovisión del personaje.

En la muestra analizada los dormitorios son mundos, con sus lógicas de funcionamiento y espacios para la aventura, aprovechando las dimensiones de algunos personajes. En Toy Story, la habitación de Andy es el hogar de Woody y sus amigos, mientras que el dormitorio de Sid es el rincón oscuro en el que esconde una imaginación turbulenta, dentro de una casa familiar promedio. Cada una de estas habitaciones, como señala Paik 
(2009), simbolizan respectivamente el cielo y el infierno para un juguete, de manera que se expanden más allá de sus dimensiones materiales.

Por otra parte, las estancias privadas cumplen la función de mostrar momentos íntimos del personaje, que revelan lo que no comparte con el resto de los habitantes de la casa, como ocurre en el hogar de los Parr (The Incredibles): Bob, el padre, construye en su estudio un refugio en el cual mitigar su frustración, atesorando sus recuerdos de glorias pasadas. Si se compara con otros espacios de la vivienda, es posible apreciar que este es un lugar para él, y que no es reflejo de incomodidad alguna con otros espacios de la edificación o problemas con su familia. Asimismo, el cuarto desnudo de Riley, en Inside Out, es una muestra patente de las dificultades de la chica para sentir propia su nueva residencia en San Francisco y no tanto, un conflicto de base con sus padres.

\section{b. Objetos}

En relación a los objetos, la muestra analizada, arroja que más allá de su función como ornamento del escenario, su presencia en las casas dota de verosimilitud a la historia, personalizando el ambiente, contribuyendo a la narración y a la creación del tono del relato en esos espacios. Ellos son destacados por el narrador para apuntar algo o para dotarlo de sentido a lo largo de la trama.

En su mayoría, los objetos estudiados marcan el avance del tiempo, como las paredes de las habitaciones infantiles que sirven de residencia a Woody y su pandilla en Toy Story, gracias a cambios en el papel tapiz y al uso de posters, se trazan las distintas etapas de los niños y de los juguetes, así como la emoción que guiará el relato, aun cuando la acción se desarrolle fuera de sus dominios. En esta saga en especial, un gesto cotidiano como cambiar un edredón por otro de motivo distinto, puede representar un giro drástico en la vida del protagonista.

Por otra parte, los objetos decorativos se focalizan más o menos, con la finalidad de reforzar el conocimiento del personaje y sus afectos. Inclusive aquellas cosas, un tanto ocultas en los fondos del escenario, cumplen con mostrar la dimensión emocional del personaje como ocurre, por ejemplo, en el apartamento de Mike en Monsters, Inc., en cuyo salón se ubica la fotografía de Celia, su novia, sin que el portarretrato sea llevado a un primer término de la imagen. De igual forma el osito de peluche que tiene el ciclope en su casa, se convierte en indicio de un rasgo infantil del personaje y la anticipación del poder energético de la risa sobre el del llanto (PérezGuerrero, 2011).

Otro de los usos, encontrados en las películas analizadas tienen fines más poéticos, como la bombilla que Wall-E saca de su colección de cosas viejas y entrega a Eva, quien la enciende solo con tocarla, convirtiendo esa acción en un símil de cómo ante los ojos del amante, el amado hace bellas y nuevas todas las cosas.

Por último, se pueden identificar una red de símbolos muy sólida, especialmente en lo que se refiere a objetos significativos para la trama, en términos de Rosenfeld (2008), con los que se apunta a cuestiones centrales del tema de cada historia y que viajan de las casas al exterior de ellas, alargando su influjo en la historia. De este modo, una simple tapa de botella se carga de sentido hasta constituirse en la acreditación de la pertenencia a una familia $(U p)$, o la rasgadura del tapiz bordado por una madre, en el que se representa a la familia, a manos de su hija, puede significar una herida que afecte a todo un reino, como ocurre en Brave.

Espacios exteriores de pertenencia: un 


\section{planeta, una ciudad, un barrio...}

Desde Homero hasta la actualidad las narraciones que trazan el retorno al hogar nos remiten a la patria: "uno de los conceptos más abstractos, míticos y luchados de entre las nociones asociadas al hogar" (Naficy, 1999, p. 6). La patria, en un sentido básico, es el lugar en el que se nace y en donde se establecen los primeros afectos: la familia, los amigos, un paisaje, -sus aromas y sonidos-, el idioma en el que se designa el mundo, la idiosincrasia.

En este tipo de espacios dramáticos, lo primero que llama la atención, en los filmes analizados, es la relación estrecha que tienen los protagonistas con sus lugares de origen. Son varias las escenas en las que se aprecia el contraste entre las diversas procedencias de las figuras del relato, como la sorpresa de Matter ante la sofisticación tecnológica de Japón y su añoranza de Radiator Springs en Cars 2 o cuando, en Toy Story, los juguetes de Andy se presentan a Buzz y hablan de sus marcas, como si se tratara de su familia y de su país de nacimiento, un aspecto fundamental de su identidad.

Desde el punto de vista de la representación de estos lugares, el análisis revela que, si en las viviendas de Pixar destaca el uso de objetos y de estancias, en la ciudad brilla el ingenio con el que edificios y calles son diseñados; el empleo de figuras retóricas para mostrar un terruño atractivo, así como el uso de elementos urbanos que, en metrópolis fantásticas, evocan urbes globalmente reconocidas, como Nueva York (Metroville), un pueblo abandonado de la Ruta 62 (Radiator Spring) o una ciudad media estadounidense (Monstruopolis, New Mushroomton).

Así mismo, se aprecia un alto nivel de detalles a la hora de trazar la recreación de lugares reales, como París (Ratatouille), la Gran Sabana venezolana (Up); San Francisco (Inside Out), Nueva York (Soul), Porto Rosso (Luca) o aquellos sitios más indeterminados, pero igualmente reconocibles, por su vinculación cultural a países como México (Coco) o Irlanda (Brave). Por tanto, puede afirmarse que en todos los casos estos espacios están "dotados de propiedades específicas" (Casetti \& DiChio, 1996, p. 177), están caracterizados y son ricos en detalles, como se aprecia en la tabla 2.

Tabla 2.

Espacios exteriores asociados al hogar ${ }^{7}$

\begin{tabular}{|c|c|c|c|c|c|}
\hline Título & Lugar & D & $\mathbf{A}$ & $\mathbf{P}$ & $\mathbf{S}$ \\
\hline Toy Story 1 & 234 Elm St. & $\mathrm{P}$ & A & $\mathrm{M}$ & $\mathrm{T}$ \\
\hline A Bug's life & Isla hormiga & $\mathrm{R}$ & A & $\mathrm{M}$ & $\mathrm{C}$ \\
\hline Toy Story 2 & 234 Elm St. & $\mathrm{P}$ & A & M & $\mathrm{T}$ \\
\hline Monsters, Inc & Monstruopolis & $\mathrm{R}$ & A & $\mathrm{M}$ & $\mathrm{T}$ \\
\hline $\begin{array}{l}\text { Finding } \\
\text { Nemo }\end{array}$ & Anémona & $\mathrm{R}$ & A & $\mathrm{M}$ & $\mathrm{T}$ \\
\hline $\begin{array}{l}\text { The Incredi- } \\
\text { bles }\end{array}$ & Metroville & $\mathrm{R}$ & A & $\mathrm{M}$ & $\mathrm{C}$ \\
\hline Cars & $\begin{array}{l}\text { Circuito/ } \\
\text { Radiator } \\
\text { Spring } \\
\end{array}$ & $\mathrm{R}$ & A & $\mathrm{M}$ & $\mathrm{C}$ \\
\hline Ratatouille & $\begin{array}{l}\text { Campiña / } \\
\text { Paris }\end{array}$ & $\mathrm{R}$ & A & M & $\mathrm{C}$ \\
\hline Wall-E & Planeta tierra & $\mathrm{R}$ & $\mathrm{D}$ & M & $\mathrm{C}$ \\
\hline$U p$ & San Francisco & $\mathrm{R}$ & D & M & $\mathrm{C}$ \\
\hline Toy Story 3 & $\begin{array}{l}234 \text { Elm St./ } \\
1225 \text { Sycamor } \\
\text { St. } \\
\end{array}$ & $\mathrm{R}$ & A & $\mathrm{M}$ & $\mathrm{T}$ \\
\hline Cars 2 & $\begin{array}{l}\text { Radiator } \\
\text { Spring }\end{array}$ & $\mathrm{R}$ & $\mathrm{D}$ & M & $\mathrm{C}$ \\
\hline Brave & Irlanda & $\mathrm{R}$ & A & $\mathrm{H}$ & $\mathrm{C}$ \\
\hline $\begin{array}{l}\text { Monsters } \\
\text { University }\end{array}$ & Monstruopolis & $\mathrm{R}$ & D & M & $\mathrm{C}$ \\
\hline Inside Out & $\begin{array}{l}\text { San Francisco } \\
\text { Minnesota }\end{array}$ & $\mathrm{R}$ & A & M & $\mathrm{C}$ \\
\hline $\begin{array}{l}\text { The Good } \\
\text { Dinosaur }\end{array}$ & Tierra & $\mathrm{R}$ & A & $\mathrm{H}$ & $\mathrm{C}$ \\
\hline
\end{tabular}




\begin{tabular}{|c|c|c|c|c|c|}
\hline Finding Dory & $\begin{array}{l}\text { California/ } \\
\text { Anémona }\end{array}$ & $\mathrm{R}$ & A & $\mathrm{M}$ & $\mathrm{C}$ \\
\hline Cars 3 & $\begin{array}{l}\text { Radiator } \\
\text { Spring/ } \\
\text { Circuito de } \\
\text { carreras }\end{array}$ & $\mathrm{R}$ & A & $\mathrm{M}$ & $\mathrm{C}$ \\
\hline $\mathrm{Coco}$ & México & $\mathrm{R}$ & A & $\mathrm{M}$ & $\mathrm{C}$ \\
\hline $\begin{array}{l}\text { The Incredi- } \\
\text { bles } 2\end{array}$ & Metroville & $\mathrm{R}$ & A & $\mathrm{M}$ & $\mathrm{C}$ \\
\hline Toy Story 4 & $\begin{array}{l}1225 \text { Sycamo- } \\
\text { re St. }\end{array}$ & $\mathrm{R}$ & A & $\mathrm{M}$ & $\mathrm{T}$ \\
\hline Onward & $\begin{array}{l}\text { New Mus- } \\
\text { hroomton }\end{array}$ & $\mathrm{R}$ & A & $\mathrm{M}$ & $\mathrm{C}$ \\
\hline Soul & Nueva York & $\mathrm{R}$ & A & $\mathrm{M}$ & $\mathrm{C}$ \\
\hline Luca & $\begin{array}{l}\text { Porto Rosso/ } \\
\text { fondo del mar }\end{array}$ & $\mathrm{R}$ & A & $\mathrm{M}$ & $\mathrm{C}$ \\
\hline
\end{tabular}

Nota: elaboración propia.

Sin embargo, hay que hacer la salvedad, de que cuando se trata de calles, la representación de espacios exteriores resulta más bien típica, sobre todo, si se compara con la importancia que los relatos otorgan al interior de las viviendas, mediante criterios anagráficos y de focalización.

Las únicas excepciones a este respecto son la anémona de Finding Nemo y Finding Dory, así como las dependencias de Isla Hormiga, frente a su exterior. No obstante, en estos filmes, los principios de armonía tienden a hacer de sus universos orgánicos y estéticamente hermosos.

En los títulos en los que presentan disarmonías en formas y espacios, son aquellos en los que los contrastes subrayan el nivel del conflicto, como en $U p$, en el que las viviendas del personaje (casa y dirigible) chocan con la modernidad de la ciudad, mediante sus formas y usos. Del mismo modo, en Wall-E, el desértico ambiente de la tierra contrasta con los espacios coloridos, aunque artificiales, de la nave Axiom, solo siendo unidos por el alegre verde de la planta que detona y concluye la trama.

Finalmente, en lo que se refiere al periodo en que se sitúan las historias, se halla que todas tienen un carácter metahistórico; es decir que no es posible precisar un año concreto, cuando menos se puede señalar una época, como la prehistoria (The Good Dinosaur) o la Edad Media (Brave).

\section{El hogar en la trama}

En un sentido más abstracto, la patria se identifica con el mundo ordinario de la historia, a menos que el héroe halle su auténtica morada en el mundo extraordinario, gracias al encuentro con nuevas personas o el descubrimiento de una pasión. Es en este punto en el que se comprueba que un hogar no es una casa ni tampoco, necesariamente, un lugar de nacimiento.

De los veinticinco filmes de Pixar estudiados, en diecinueve de ellos, los protagonistas encuentran su hogar en el mundo ordinario: Woody en Toy Story 1 y 2; Flik, en A Bugs'Life; Sulley y Mike en las entregas de Monsters, Inc; Marlin y Nemo en Finding Nemo, así como junto a Dory, en Finding Dory; Los Parr en The Incredibles; Mater en Cars 2; Rayo McQueen en Cars 3, Merida en Brave; Miguel en Coco, Wall-E, en el film homónimo; Alegría y Tristeza en Inside Out, Carl en Up, Ian y Barley Lightfoot en Onward; Joe en Soul y Arlo en The Good Dinasaur.

Por otra parte, en ningún caso los protagonistas de Pixar renuncian al hogar hallado en el mundo extraordinario. Por el contrario, cuatro héroes de los títulos estudiados, reconocen y abrazan el hogar que este territorio les brinda: Rayo McQueen (Cars), Remi (Ratatouille), Woody y su pandilla en Toy Story 3 y Luca. La excepción a esta tendencia es Woody, en Toy Story 4, quien renuncia al hogar recuperado en el mundo ordinario, para marcharse con Bob Peep, su interés romántico. 
Es interesante notar como este resultado, aunque muestra dos tendencias, en realidad, responde a una sola: el hogar es donde está el corazón, y pese a que los personajes muestran un amor profundo por sus ciudades o lugares de origen, hay dos elementos a los que no renuncian, ya que los definen por encima de todo: la familia y el trabajo, entendido como pasión.

En todas las tramas en las que el hogar está en el mundo ordinario, los personajes salen al mundo extraordinario junto a sus amigos. Conviene recordar que todas las películas de Pixar son historias de compañeros. Es así como en su regreso a casa, las figuras principales del relato regresan con quienes moraban en el mundo ordinario y compartieron su aventura. Además, varios de estos protagonistas, traen consigo a los personajes que han adoptado como familia, lo que hace mejor su hogar.

Un camino que es muy similar al que transitan aquellos que encuentran su morada en el mundo extraordinario, ya que estos personajes suelen traer, o atraer, a sus familias a este nuevo territorio, conjugando así, sus ambiciones profesionales y vitales con la convivencia familiar. Es el caso de Rayo McQueen (Cars), cuyo hogar está en el circuito de carreras, pero sus amigos de Radiator Spring lo acompañan o Remi (Ratatouille), quien recupera a su familia en París y logra convertirse en el mejor chef de la ciudad. Por su parte, Woody y su pandilla, en Toy Story 3, siguen al servicio de un nuevo niño, pero se mantienen juntos como familia. Finalmente, Luca, consigue construir con su familia y amigos un hogar al cual volver, mientras se encuentra estudiando en

Génova.

\section{DISCUSIÓN}

\section{La familia, como lugar de encuentro y forja de identidad}

En los films estudiados, la asociación entre familia y hogar es tan fuerte que sus sentidos resultan intercambiables. Sin embargo, hay que notar que la noción de familia a la que se refieren estas películas se halla dentro de la tendencia de buena parte de la filmografía mainstream estadounidense actual, especialmente del cine familiar, en la que "la familia no es simplemente la entidad biológica de la familia nuclear, sino más bien un grupo de individuos que se cuidan unos a otros, estén o no técnicamente relacionados [...] una red afectiva de amplio espectro" (Tyler, 2013, p. 269).

En tal sentido, puede decirse que esta "familia extendida" sigue estando asociada a la noción, un tanto idealizada, de comunidad fundada en el amor desinteresado y en el respeto entre sus integrantes; una concepción que como Alexander indica (2013), ha sido revisada por los relatos a lo largo de los siglos, y que constituye un eficaz agente de cambio ético en el marco de la cultura narrativa. En consecuencia, dentro los patrones posibles de convivencia, pareciera que Pixar encuentra en esta apertura y afecto familiar un modelo idóneo de colectividad que propone como ideal social, que se remarca al contraponer el estilo de vida de sus héroes a la de sus villanos, quienes suelen vivir en soledad y si viven con otros, sus relaciones no se basan en la confianza.

La asociación entre seres queridos y hogar, se revela en cada aspecto de las cintas analizadas. Es así como, pese a que la representación material de las casas se acerca al tópico de locus amoenus ${ }^{8}$, el valor de estas edificaciones se esfuma cuando los seres queridos del protagonista ya no viven en ella o cuando hay que extender la familia a otros miembros (amigos). Por ejemplo, en el clímax de The

8 Tópico literario que tiene su origen en Homero y que cobró mayor relevancia durante la Edad Media. Consiste en la descripción de un lugar idílico, donde suele hâber prado, sombra, aves canoras, un arroyo cristalino. En la actualidad, el tópico tiene vigencia. El cine acude a él cuando habla de paisajes recordados y soñados. Este concepto se puede aplicar también a personas (personajes). 
Incredible 1, la familia de superhéroes da muy poca importancia a la pérdida total de su morada, mientras celebra la unión renovada entre ellos. En Toy Story 1, Woody y Buzz encuentran su felicidad al alcanzar el auto de Andy y vivir con él en su nuevo domicilio. Asimismo, en la tercera entrega de la saga, cuando los muñecos se mudan a la casa de Bonnie y se integran a la comunidad de juguetes de ese dormitorio, sus sentimientos son de alegría pues volverán a estar vivos en la imaginación infantil, podrán servir a la niña hasta que los necesite, y conservarán el amor a Andy, como parte de su hogar, en sus corazones y recuerdos.

$\mathrm{Y}$ es que vale la pena mencionar que, en el análisis, se evidenció la impronta que deja en los personajes la pertenencia ineluctable al núcleo familiar, como aspecto constitutivo de su identidad. Es decir, cuando el personaje de los filmes estudiado debe dejar de ver a algún ser querido o abandonar su lugar de residencia habitual, casi siempre motivado por razones altruistas, no puede ni quiere renunciar a los vínculos que le unen a esas personas y lugares, por lo que apela siempre a ese ámbito personal e intangible del afecto y del recuerdo, manteniendo la integridad de su vínculo con aquellos que ama, por lo que ellos han significado en la constitución de su modo de ver el mundo.

En $U p$, por ejemplo, cuando Carl suelta su casa sobre las Cataratas Paraíso experimenta un encuentro más pleno con Ellie, al reconectar con los valores que aprendió y vivió con ella: “acogimiento, entusiasmo, compañerismo, lealtad, deseos de aventura" (Perez-Guerrero, 2011, p. 366).

En Monsters, Inc., Sulley recupera el hogar, tras renunciar a él por el bien de todos, cuando Mike reconstruye la puerta que lo lleva a la habitación de Boo, a ese rincón en el que el personaje encuentra razones para ser mejor, a través de los sentimientos paternales que profesa a la pequeña.

Así, estas narrativas referidas al hogar coinciden con otras que "están inscritas en la nostalgia de un sentido de pertenencia no contaminado" (Bonfren, 2004, p. 22). Una añoranza que apela a reconocer y apreciar la propia identidad, adquirida en y por la familia, como motivación esencial de desarrollo personal y social del héroe (Brave, Toy Story, A Bugs Life, Cars, Monsters, Up), y a la que le resulta imposible renunciar, como ocurre a Dory en este diálogo de Finding Nemo:

\section{DORY}

...Por favor, no te vayas. Por favor. Nadie había aguantado tanto tiempo conmigo. Y si te vas... Y si te vas...

(trata de retenerlo)

Yo solo, recuerdo mejor las cosas ¡Escucha!

(Canturreando)

P. Sherman, calle Wallaby cuarenta y... dos ¡Oh!

(No puede recordar; desesperada)

Lo sé, me acuerdo porque... cuando te miro, lo siento, Y cuando te miro, estoy... como en casa.

No hay respuesta.

DORY

Por favor. No quiero perder esto. No quiero olvidar (Stanton, Peterson \& Reinolds, 2002, p. 124).

Tabla 3.

El hogar en las tramas de Pixar

\begin{tabular}{|c|l|l|l|}
\hline Título & \multicolumn{1}{|c|}{$\begin{array}{c}\text { Mundo ordi- } \\
\text { nario }\end{array}$} & $\begin{array}{l}\text { Hogar defini- } \\
\text { tivo }\end{array}$ & $\begin{array}{l}\text { Resul- } \\
\text { tado }\end{array}$ \\
\hline Toy Story 1 & Cuarto de Andy & Cuarto de Andy & $\begin{array}{l}\text { Andy/Ami- } \\
\text { gos }\end{array}$ \\
\hline
\end{tabular}




\begin{tabular}{|c|c|c|c|}
\hline A Bug's life & Isla hormiga & Isla hormiga & $\begin{array}{l}\text { Comunida- } \\
\text { d/I. Románti- } \\
\text { co/amigos }\end{array}$ \\
\hline Toy Story 2 & Cuarto de Andy & Cuarto de Andy & $\begin{array}{l}\text { Andy/Ami- } \\
\text { gos }\end{array}$ \\
\hline $\begin{array}{l}\text { Monsters, } \\
\text { Inc. }\end{array}$ & Fábrica de gritos & $\begin{array}{l}\text { Fábrica de risas/ } \\
\text { cuarto de Boo }\end{array}$ & $\begin{array}{l}\text { Trabajo/ } \\
\text { amigos }\end{array}$ \\
\hline $\begin{array}{l}\text { Finding } \\
\text { Nemo }\end{array}$ & Anémona & Anémona & $\begin{array}{l}\text { Anémona/ } \\
\text { amigos }\end{array}$ \\
\hline $\begin{array}{l}\text { The Incre- } \\
\text { dibles }\end{array}$ & Familia & Familia & Familia \\
\hline Cars & Circuito & Radiator Spring & $\begin{array}{l}\text { Trabajo/ } \\
\text { amigos }\end{array}$ \\
\hline Ratatouille & $\begin{array}{l}\text { Campiña france- } \\
\text { sa/cocina) París }\end{array}$ & $\begin{array}{l}\text { París/ balsa/res- } \\
\text { taurant }\end{array}$ & $\begin{array}{l}\text { Trabajo/ } \\
\text { amigos }\end{array}$ \\
\hline Wall-E & Planeta tierra & Planeta tierra & $\begin{array}{l}\text { Trabajo/ } \\
\text { Amigos }\end{array}$ \\
\hline$U p$ & $\begin{array}{l}\text { Casa/San Fran- } \\
\text { cisco }\end{array}$ & Zepelling/San F. & Amigos \\
\hline Toy Story 3 & Cuarto de Andy & $\begin{array}{l}\text { Cuarto de Bon- } \\
\text { nie }\end{array}$ & $\begin{array}{l}\text { Trabajo/ } \\
\text { amigos }\end{array}$ \\
\hline Cars 2 & Radiator Spring & Radiator Spring & Amigos \\
\hline Brave & Reino & Reino & $\begin{array}{l}\text { Familia/co- } \\
\text { munidad }\end{array}$ \\
\hline $\begin{array}{l}\text { Mosnters } \\
\text { University }\end{array}$ & Fábrica de gritos & Fábrica de gritos & $\begin{array}{l}\text { Trabajo/ } \\
\text { amigos }\end{array}$ \\
\hline Inside Out & Familia & Familia & Familia \\
\hline $\begin{array}{l}\text { The Good } \\
\text { Dinosaur }\end{array}$ & Granja & Granja & $\begin{array}{l}\text { Familia/ami- } \\
\text { gos }\end{array}$ \\
\hline \begin{tabular}{|l} 
Finding \\
Dory
\end{tabular} & Arrecife & Arrecife & $\begin{array}{l}\text { Familia/ami- } \\
\text { gos }\end{array}$ \\
\hline Cars 3 & $\begin{array}{l}\text { Radiator Spring/ } \\
\text { Circuito de ca- } \\
\text { rreras }\end{array}$ & $\begin{array}{l}\text { Circuito de ca- } \\
\text { rreras }\end{array}$ & $\begin{array}{l}\text { Familia/ami- } \\
\text { gos }\end{array}$ \\
\hline Coco & Casa de Miguel & Casa de Miguel & $\begin{array}{l}\text { Familia/ami- } \\
\text { gos }\end{array}$ \\
\hline $\begin{array}{l}\text { The Incre- } \\
\text { dibles } 2\end{array}$ & Familia & Familia & $\begin{array}{l}\text { Familia/ami- } \\
\text { gos }\end{array}$ \\
\hline Toy Story 4 & $\begin{array}{l}234 \text { Elm Street/ } \\
1225 \text { Sycamore } \\
\text { St. }\end{array}$ & Nuevos lugares & $\begin{array}{l}\text { Interés ro- } \\
\text { mántico }\end{array}$ \\
\hline \multirow[t]{2}{*}{ Onward } & $\begin{array}{l}\text { New Mus- } \\
\text { hroomton/ }\end{array}$ & $\begin{array}{l}\text { New Mu- } \\
\text { chroomton/ }\end{array}$ & $\begin{array}{l}\text { Familia/ } \\
\text { Amigos/ }\end{array}$ \\
\hline & Casa & Instituto & Comunidad \\
\hline Soul & Nueva York & Nueva York & $\begin{array}{l}\text { Individuo } \\
\text { pleno }\end{array}$ \\
\hline Luca & $\begin{array}{l}\text { Fondo del mar / } \\
\text { Portorosso }\end{array}$ & Génova & $\begin{array}{l}\text { Familia/ami- } \\
\text { gos/ } \\
\text { Individuo }\end{array}$ \\
\hline
\end{tabular}

Nota: elaboración propia.

\section{La patria, entre la comunidad y el territorio}

La patria, entendida como lugar de nacimiento o el lugar en el que se vive, muestra en los filmes estudiados, valores de comunidad que se acercan a lo expresado en el análisis hecho a las viviendas de los protagonistas. La muestra de ello es la caracterización de las ciudades, frente a lo típico de las calles. Al fin y al cabo, un diseño rico y reconocible ofrece razones para entender la relación que tienen los personajes con su comunidad.

En tal sentido, es preciso señalar que varios de los protagonistas ocupan un puesto de liderazgo en sus ciudades o lo logran tras finalizar la aventura, mediante la aportación de algún valor. De hecho, en la fase de retorno al hogar, el elixir con que el héroe de Pixar regresa a la patria no es otro que su perfeccionamiento personal.

Tras la superación de su error fatídico durante la aventura, viene a favorecer a su pueblo con las virtudes adquiridas, bien para defenderla del mal (The Incredibles 1 y2, A Bug's Life) o para ayudar a sus paisanos a crear una mejor sociedad (Toy Story,1,2 y 3; Finding Nemo y Dory; Monsters, Inc., Cars, Ratatouille, Wall-E Brave, Coco, Onward, Luca). Además, no solo vendrá con nuevas cualidades, sino que también contribuye con nuevos miembros, con los cuales trabajar en pos del futuro colectivo. Y es que en todas las historias estudiadas los protagonistas apuestan por la posibilidad de bondad y participación valiosa de cada una de las figuras que pueblan sus relatos; incluso de los villanos o fuerzas antagonistas, siempre y cuando acepten esta alternativa.

Esta postura vital de los personajes pixarianos es lo que James Clark (2013) denomina, romanticismo americano, la cual consiste en una actitud ante la vida, consciente de los demás, cuyo resultado es el crecimiento espiritual e intelectual del protagonista y que se ha convertido en una tendencia en el cine de Estados Unidos actual. 
Si bien es cierto que el carácter idílico y nostálgico que representa la narrativa de Pixar es evidente, también es verdad que no por eso rehúye a una mirada crítica a la sociedad de hoy, denunciando efectos sociales de la mezquindad humana. En todos los terruños de sus historias es posible reconocer problemas que aquejan al mundo actual: "el hedonismo (Wall-E), la contaminación ambiental (Monsters, Inc., Wall-E), la exacerbación de la lucha por ciertos 'derechos' (The Incredibles), la frívola persecución del éxito y la velocidad de la vida (Cars), la dominación de los más fuertes sobre los débiles (A Bug's Life), la intolerancia hacia los diferentes" (Pérez-Guerrero 2014, p. 83), como en Ratatouille y Luca.

Tal vez, por eso, en este rasgo se pueda reconocer una de las razones narrativas de los creativos del Estudio para la caracterización de sus espacios exteriores, además del solo espectáculo. Y es que, la ambientación en la que se desarrollan estas historias es altamente conflictiva y los asuntos que se han mencionado no son marginales en el argumento, sino que ocasionan tensiones en esas comunidades y plantean desafíos éticos a sus protagonistas, por lo que requieren de un mayor grado de detalle y personalización.

Por tanto, la salida que se propone en estos filmes, centrada en una mejora colectiva, a partir del perfeccionamiento personal por otros, aunque puede parecer contradictoria para algunos (Sperb, 2015), no es ingenua, se aleja de cinismos y se acerca a una posibilidad de cambio, al menos, al alcance de cada uno.

Finalmente, se puede decir que la patria, entendida como ciudad o lugar de nacimiento, representa exactamente lo que son: territorio, comunidad y sentido de pertenencia a ellos. En ese sentido, la película de Wall-E expande el espacio y la responsabilidad de cada uno para que el planeta sane. Un valor que, sin duda, no es reprochable ni sospechoso, sino más bien, adecuado a una sociedad como la de hoy y un público como el familiar.

\section{CONCLUSIONES}

Los usos creativos que se dan al hogar en las producciones de Pixar, le otorgan un lugar singular. Desde el punto de vista narrativo, en la mayoría de los casos, recae una función actacial de ayudante, de objeto o de destinatario, lo cual viene acompañado de una estética atractiva, reconocible y altamente significativa.

Si bien, la idea de equiparar el hogar a la familia como una comunidad abierta a los amigos no es para nada original, lo que hace especial este tratamiento del hogar es el hecho de que la aborden como una realidad que cambia, dando una mayor importancia al vínculo genuino de amor entre sus miembros, que a una definición sobre el tipo de familia que están representando.

Por otra parte, desde un punto de vista empresarial, esta opción creativa permite a los filmes resistir mejor el paso del tiempo y algunos cambios de mentalidad, lo cual es subrayado por la dimensión individual de sus personajes (peces, juguetes, monstruos marinos), a lo que contribuye el hecho de inscribirse en el género de fantasía de aventuras y ser realizado en animación.

No obstante, lo anterior, desde la perspectiva de este trabajo, lo que más importa es la idea de la familia y la amistad como factores que humanizan las sociedades, baluartes de lo auténticamente humano; aunque el trabajo, entendido como pasión y camino de servicio a otros, se alce como otro valor al cual sus personajes tienden de manera radical, convirtiéndolo 
en ingrediente indispensable de la receta que Pixar entiende como hogar.

\section{BIBLIOGRAFÍA}

Alexander, L. A. (2013). Fictional Words I: The Symbolic Journey \& The Genre System. Createspace.

Bachelard, G. (1994). The Poetics of Space, The Classic Look at How We Experience Intimate Place. Beacon.

Block, B. (2008), The Visual Story, Focal press.

Bronfen, E. (2004), Home in Hollywood, The Imaginary Geography of Cinema, Columbia University Press.

Campbell, J. (1993). The Hero with a Thousand Faces, Fontana Press.

Casetti, F. \& Di Chío, F. (1996). Cómo analizar un film, Paidós.

Cauquelin, A. (2007). A Invençao da Paisagem. Livraria Martins Fontes Editora Ltd.

Cheu, J. (2013). Diversity in Disney Films Critical Essays on Race, Ethnicity, Gender, Sexuality and Disability, McFarlan.

Clark, J. (2013). The Films of Pixar Animation Studio, Kamera Books.

Heidegger, M. (1989). Construir, morar, pensar. Revista Camacol, 12 (2) 148-151.

Holliday, C. (2016). Carl's Moving Castle, “Animated" Houses and the Renovation of Play in Up (2009). En: Andrews, E, Hockenhull, S. y Pheasant-Kelly, F. (2016). Spaces of the Cinematic Home, Behind the Screen Door. Routledge.

Jenkings, (2015). Home movies, The American Family in Contemporary Hollywood Cinema, Tauris Academic Studies.

Kuntz, M. (1993). Narrative Setting and Dramatic Poetry. E. J. Brill.

Lefevre, M. (2006). Landscape and Film. Afi Film Readers.

Meinel, D. (2014). Empire is out There!?: The spirit of imperialism in the Pixar animated film Up. NECSUS. European Journal of Media Studies, Jg. 3 Nr. 1, S. 69-87. DOI: https:// doi.org/10.25969/mediarep/15135.

Naficy, H. (ed.) (1999). Home, Exile, Homeland,
Film, Media, and the Politic of Place. Routledge.

Paik, K. (2007). To infinity and Beyond. Virgin Books.

Pérez-Guerrero, A. M. (2011). Estrategias narrativas orientadas a la construcción de niveles de lectura en el cine de animación de Pixar (1995-2010). Tesis doctoral. Universidad Rey Juan Carlos.

Pérez-Guerrero, A. M. (2014). El heroísmo cotidiano en los filmes de Pixar. En Gutiérrez de Terán, J, \& Gutiérrez Carreras, P. (Eds). Hombre y Dios en el cine contemporáneo, CEU Ediciones, pp. 77-85.

Price, D. (2008). The Pixar Touch, The Making of a Company, Alfred A. Knopp.

Prokhorov, A. (2021). The Hero's Journey and Three Types of Metaphor in Pixar Animation, Metaphor and Symbol, 36 (4), pp. 1-12, doi: 10.1080/10926488.2021.1919490

Rosenfeld, J. (2008). Make a Scene, Crafting a Powerful Story One Scene at a Time, FW Publications.

Sánchez-Escalonilla, A. (2009). Fantasías de aventuras. Ariel.

Sperb, J. (2015). Flickers of Film Nostalgia in the Time of Digital Cinema, Rutgers University Press, pp. 89-113.

Stanton, A, Peterson, B \& Reinolds, D. (2002). Finding Nemo, Guion original, Pixar Animation Studios.

Tayler, D. (2013). Home is Where the Heart is, Pixar's Up. Diversity in Disney Film, Critical Essay on Race, Ethnicity, Gender, Sexuality and Disability. Cheu, J (Ed.). McFarland, 268-283.

Treimblay, P. (2011). Home Sweet Home: The Cautionary Prison/Fairytale. Wide Screen. No1(3).

Ubersfeld, A. (1993). Semiótica teatral, Cátedra. Vogler, C. (2007), The Writer's Journey: Mythic Structures for Writers. Michael W 